\title{
Stratospheric control of planetary waves
}

${ }^{1}$ Department of Applied Mathematics and Theoretical Physics, University of Cambridge, Cambridge, UK.

\section{Key Points:}

- Coupled evolution of the stratospheric mean state and planetary waves drives half of their amplification prior to sudden warmings

- Imposing sudden-warming-like anomalies creates a downward-migrating region of local wave mean-flow interaction confined to the stratosphere

- The dependence of the equatorward shift of the tropospheric jet on the height of the imposed anomalies is quantified

${ }^{*}$ Current address, National Center for Atmospheric Research, 3450 Mitchell Lane, Boulder, CO, USA

Corresponding author: Peter Hitchcock, phitch@ucar .edu 


\section{Abstract}

The effects of imposing at various altitudes in the stratosphere zonally symmetric circulation anomalies associated with a stratospheric sudden warming are investigated in a mechanistic circulation model. A shift of the tropospheric jet is found even when the anomalies are imposed only above $2 \mathrm{hPa}$. Their influence is communicated downwards through the planetary wave field via three distinct mechanisms. First, a significant fraction of the amplification of the upward fluxes of wave activity prior to the central date of the warming is due to the coupled evolution of the stratospheric zonal mean state and the wave field throughout the column. Second, a downward-propagating region of localized wave, meanflow interaction is active around the central date, but does not penetrate the tropopause. Third, there is deep, vertically synchronous suppression of upward fluxes following the central date. The magnitude of this suppression correlates with that of the tropospheric jet shift.

\section{Introduction}

The influence of the stratosphere on surface weather and climate is of interest not only for possible associated gains in medium-range to seasonal forecasting [Sigmond et al., 2013; Scaife et al., 2015] and for its role in a changing circulation resulting from changing greenhouse gases [Manzini et al., 2014], but also because there are pathways to surface impacts for a variety of specific middle atmosphere forcings, including solar forcing [Kodera and Kuroda, 2002; Ineson et al., 2011], volcanic eruptions [Muthers et al., 2014], and the quasibiennial oscillation [Gray et al., 2004]. The mechanisms invoked often include a connection from the forcing to the occurrence of stratospheric sudden warmings (or other dynamical behaviour of the polar vortex) and from there to surface impacts.

There is very clear evidence that forcing in the lower stratosphere does influence the tropospheric circulation. The strongest case in observations arises from the Antarctic ozone hole, which is believed to have led to an observed poleward shift of the Southern Hemisphere surface westerlies [see Previdi and Polvani, 2014, for a recent review]. Modeling studies have shown that imposing similar anomalies in the Arctic polar vortex leads to a surface response as well [e.g. Douville, 2009; Hitchcock and Simpson, 2014]. In the Northern Hemisphere, one of the major motivations for these studies has arisen from considering the consequences of stratospheric sudden warmings [Baldwin and Dunkerton, 2001]. But whether a sudden warming can be considered an 'externally imposed' strato- 
spheric anomaly is not clear since warmings are driven by planetary-scale Rossby waves whose sources are predominantly tropospheric.

The strength of surface impacts from forcings higher in the stratosphere, such as solar cycle effects, is less clear. A continuing cause of confusion in this subject is the relation between observed time evolution of circulation anomalies and the existence of mechanisms for downward influence. The fact that anomalies in the upper stratosphere are often observed to precede those at lower levels does not in itself imply that the upper level anomalies are the cause of the lower level anomalies. This possibility was clearly demonstrated by Plumb and Semeniuk [2003] in an idealized model of polar stratospheric variability [Holton and Mass, 1976], where constraining the mean state above a fixed level led to little impact on the evolution of the flow below. Similarly, the descent of the stratospheric cold anomaly which follows a subset of major sudden warmings known as Polarnight Jet Oscillation (PJO) events can largely be explained by the vertical gradient in radiative timescales, again requiring no downward influence [Hitchcock et al., 2013a]. Although significant downward influence from high-altitude solar effects has been argued in several cases [Gray, 2003; Ineson et al., 2011], the strength of this influence and the relevant mechanisms remain unclear not least due to the strongly chaotic evolution of both the tropospheric jet and the stratospheric vortex.

Downward influence within the stratosphere is thought to arise through two types of pathways [Plumb and Semeniuk, 2003; Hardiman and Haynes, 2008]. The first is through the zonally symmetric circulations associated with the maintenance of a balanced state; these depend only weakly on the zonal mean state itself, and the effects decay exponentially with distance through which the downward influence extends [e.g. Haynes et al., 1991].

The second broad class relies on interactions between planetary-scale Rossby waves and the zonal mean stratospheric state, and depends strongly upon the latter. These can be local in character; one commonly invoked mechanism [Matsuno, 1971; Kodera and Kuroda, 2002; Ineson et al., 2011] involves the presence of a a critical line for quasistationary waves, or more generally a layer where winds are weak, below which there is strong dissipation of the waves. If this absorption is sufficiently local and coherent, this leads to deceleration of the zonal mean winds below the existing anomaly and thus descent of the region of absorption. However, it was argued by Plumb and Semeniuk [2003] 
that a mechanism of this type does not necessarily lead to downward influence, in the sense of downward propagation of information.

Non-local influence by the waves can arise in the presence of reflection [Perlwitz and Harnik, 2004; Shaw and Perlwitz, 2013] or resonances [Plumb, 1981; Matthewman and Esler, 2011; Albers and Birner, 2014], note however that the two mechanisms are not exclusive of each other. These mechanisms imply a significant degree of stratospheric control over the quasi-stationary waves throughout the depth of the atmosphere. While such control can be clearly demonstrated in highly idealized contexts [Coughlin and Tung, 2005], the relevance of this type of downward influence in real stratospheric sudden warmings remains an issue of current debate [Albers and Birner, 2014].

Since the troposphere-stratosphere system is highly chaotic, each of these mechanisms may appear to be relevant for particular initial conditions. It is therefore essential to quantify their 'deterministic' effects, which survive averaging over some non-trivial ensemble. We present in this paper a series of numerical experiments which demonstrate a deterministic response to stratospheric forcing and the dependence of this response on the height above which the forcing is applied.

We briefly present the model setup in section 2, providing more complete details in the appendix. Results are given in section 3, and conclusions are presented in section 4.

\section{Model Setup}

The numerical experiments are carried out with a version of the Reading Intermediate General Circulation Model (IGCM), a dry dynamical core. The model configuration is based on a modified setup of Polvani and Kushner [2002], appropriate for a perpetual midwinter configuration. Details of the numerics and relaxation temperature profile are given in the appendix.

To produce a stationary wave field, surface topography is specified as a mountain, Gaussian in latitude $\phi$ and longitude $\lambda$, centered in the Northern Hemisphere:

$$
\Phi_{s}=g h_{0} \exp \left(-\left(\frac{\phi-\phi_{0}}{\Delta \phi}\right)^{2}-\left(\frac{\lambda}{\Delta \lambda}\right)^{2}\right)
$$

The height $h$ of the mountain is $3 \mathrm{~km}$, centered at $\phi_{0}=45^{\circ} \mathrm{N}$ with $\Delta \phi=\Delta \lambda=15^{\circ}$. The stationary wave field has a stronger component of zonal wave number one than two. 
The base run is integrated for 100,000 days with the first 10,000 days discarded to remove the influence of an initial period of transient behaviour in the tropics. In the remaining 90,000 days, the model produces 465 stratospheric sudden warmings defined in terms of the reversal of the zonal mean eastward winds at $60^{\circ} \mathrm{N}$ and $10 \mathrm{hPa}$, discarding those reversals not preceded by 20 days of eastward winds.
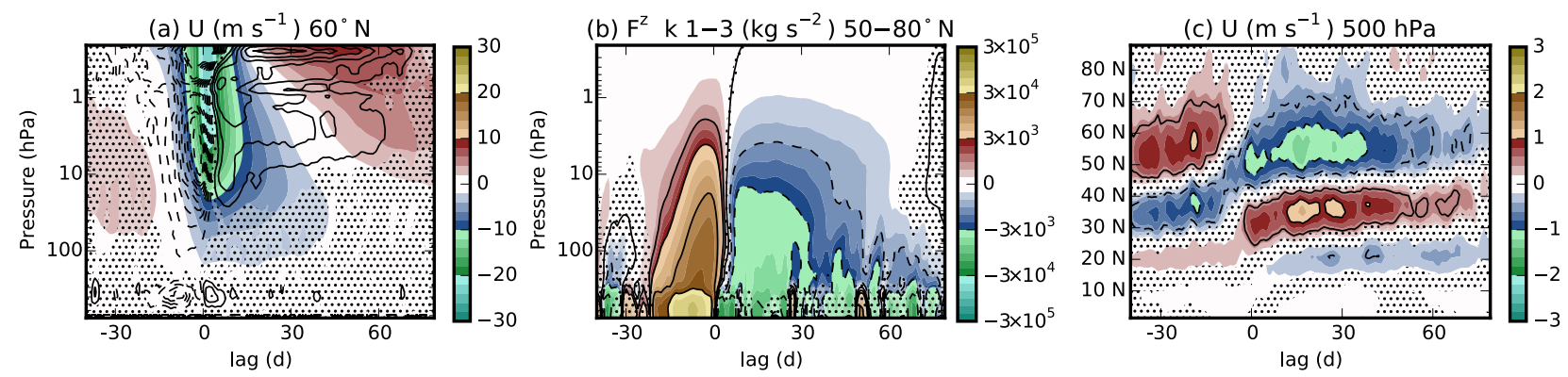

Figure 1. Composite anomalies from 465 sudden warmings in the base run. (a) Zonal mean zonal wind at $60^{\circ} \mathrm{N}$ (colors) and acceleration due to the convergence of EP flux due to planetary-scale eddies averaged from $40^{\circ}-80^{\circ} \mathrm{N}$ (contours, interval $0.5 \mathrm{~m} \mathrm{~s}^{-1} \mathrm{~d}^{-1}$ ). (b) Vertical component of the EP flux due to planetary-scale eddies averaged from $50^{\circ}-80^{\circ} \mathrm{N}$. (c) Zonal mean zonal wind at $500 \mathrm{hPa}$. Stippling indicates regions where the composite mean differs from zero at the $95 \%$ confidence interval as estimated by a t-test.

A composite over these events is constructed relative to the date of this wind reversal, which we define as the central date. The evolution of this composite, as an anomaly from the time mean, is shown in Fig. 1. Anomalous westward winds arise first in the upper stratosphere, about ten days prior to the central date. Just prior to the central date, the $2 \mathrm{~m} \mathrm{~s}^{-1}$ contour reaches $100 \mathrm{hPa}$. The lower stratospheric wind anomalies persist for about 45 days, with more rapid recovery to anomalous eastward winds in the upper stratosphere (Fig. 1a). Consistent with the standard understanding of the dynamics of these events, the wind anomalies are forced by the angular momentum transported by planetaryscale Rossby waves, as measured by the Eliassen-Palm (EP) flux due to zonal wave numbers 1 to 3 [computed following Andrews et al., 1987, from daily instantaneous output]. The upward wave fluxes amplify over two weeks prior to the wind reversal (Fig. 1b), and are subsequently suppressed until nearly 60 days after the wind reversal, consistent with composites of similar events in reanalyses and comprehensive models [Hitchcock et al., 2013b]. The anomalous divergence of these fluxes is shown in Fig. 1a, revealing a deep region of strong convergence prior to the wind reversal, followed by anomalous diver- 


\begin{tabular}{lccc}
\hline Run & Time (days) & $p_{b}$ & $p_{t}$ \\
\hline base & 100,000 & & \\
$c 1$ & 37,000 & $2 \mathrm{hPa}$ & $0.8 \mathrm{hPa}$ \\
$c 8$ & 37,000 & $10 \mathrm{hPa}$ & $6 \mathrm{hPa}$ \\
$c 30$ & 37,000 & $40 \mathrm{hPa}$ & $20 \mathrm{hPa}$ \\
$c 70$ & 37,000 & $90 \mathrm{hPa}$ & $50 \mathrm{hPa}$ \\
$s 1$ & $740 \times 160$ & $2 \mathrm{hPa}$ & $0.8 \mathrm{hPa}$ \\
$s 8$ & $740 \times 160$ & $10 \mathrm{hPa}$ & $6 \mathrm{hPa}$ \\
$s 30$ & $740 \times 160$ & $40 \mathrm{hPa}$ & $20 \mathrm{hPa}$ \\
$s 70$ & $740 \times 160$ & $90 \mathrm{hPa}$ & $50 \mathrm{hPa}$ \\
$m 30$ & $465 \times 80$ & $90 \mathrm{hPa}$ & $50 \mathrm{hPa}$ \\
$m 20$ & $465 \times 80$ & $90 \mathrm{hPa}$ & $50 \mathrm{hPa}$ \\
\hline
\end{tabular}

gence corresponding to the suppressed vertical fluxes. To a large degree these anomalies are explained by the vertical derivative of the vertical flux. During the recovery phase of the stratospheric event the tropospheric jet shifts equatorward (Fig. 1c). Significant wind anomalies are seen prior to the wind reversal in both the troposphere and stratosphere.

A series of further ensembles of integrations are then carried out following the methodology of Hitchcock and Simpson [2014, henceforth HS14]. For each ensemble, a control integration $(c 1, c 8, c 30, c 70)$ is first carried out. This is achieved by relaxing the zonally symmetric component of the circulation towards the time-averaged state of the base run ( $X_{c}$, where $X$ denotes the divergence, vorticity, or temperature). The rate of the relaxation varies linearly $(q=1)$ from $\tau_{0}=6 \mathrm{~h}$ above $p_{t}$ to zero below $p_{b}$ :

$$
K(p)=\left\{\begin{array}{l}
\tau_{0}^{-1} \text { if } p<p_{t}, \\
\tau_{0}^{-1}\left(\frac{p-p_{b}}{p_{t}-p_{b}}\right)^{q} \text { if } p_{t}<p<p_{b}, \\
0 \text { if } p>p_{b} .
\end{array}\right.
$$

The height at which this relaxation is performed is varied by setting $p_{b}$ and $p_{t}$ according to Table 1 . The region above $p_{b}$ is referred to as the nudging layer. The effects of this nudging are discussed further in the supplementary material. 
The effects of the zonally-symmetric anomalies associated with the composite sudden warming are then determined in a further set of integrations. The ensembles $(s 1, s 8$, $s 30, s 70$ ) consist of a set of 740 'nudged' integrations, initialized from the corresponding control integration at intervals of 50 days; the large ensemble size was found to be necessary to achieve a statistically robust signal, particularly in $s 1$. Each integration is carried out for 160 days, and is nudged by relaxing the circulation according to (2) towards the time-evolving composite of the sudden warmings (Fig. 1). The composite values are denoted $X_{S}(t)$, where $t$ is defined relative to the central date of the sudden warming. The reference state $X_{r}$ to which the circulation is relaxed is defined by intepolating smoothly over 10 days $\left(t_{0}\right)$ from the climatological mean to the time-varying composite, starting 40 days prior to the central date $\left(t_{s}=-40 \mathrm{~d}\right)$ :

$$
\begin{aligned}
X_{r} & =X_{c}+r\left(t, t_{0}\right)\left(X_{s}(t)-X_{c}\right), \\
r\left(t, t_{0}\right) & =\left\{\begin{array}{l}
\sin ^{2}\left(\frac{\pi}{2}\left(t-t_{s}\right) / t_{0}\right) \text { if } t-t_{s}<t_{0}, \\
1 \text { otherwise. }
\end{array}\right.
\end{aligned}
$$

The composite anomalies starting about a month prior to the wind reversal at $10^{\circ} \mathrm{N}$ $60 \mathrm{hPa}$ are thus imposed in each ensemble member through the nudging defined by (2). Since each ensemble member is initialized with an essentially random initial condition drawn from the control run, any signal in the ensemble average relative to the control integration can be interpreted as a 'deterministic' response to the imposed stratospheric anomalies, independent of the initial conditions. The responses shown below are computed by differencing the nudged integrations (comprising, e.g. s70) from the corresponding time period in the control run (e.g. $c 70$ ).

\section{Results}

Figure 2 shows the anomalies for each ensemble of the same quantities shown for the base run in Fig. 1. The nudging layer is indicated in the first and second column of panels by horizontal dashed $\left(p_{b}\right)$ and solid $\left(p_{t}\right)$ lines. In all cases the high-latitude wind response is reproduced to a good approximation within the nudging layer. When the nudging is imposed higher in the stratosphere ( $s 1, s 8$; Figs. 2a,b), wind anomalies are also produced one to two scale heights below the level of the nudging. Accompanying these anomalies is a region of EP-flux convergence which descends over time, following the wind anomalies, consistent with the local wave-mean flow interaction mechanism pro- 
posed by Matsuno [1971]. A small region of convergence is also apparent in Fig. 2c, but does not descend below $100 \mathrm{hPa}$, and no such feature is seen in Fig. 2d, suggesting this mechanism is only active within the stratosphere. Note that there are artifacts in the flux divergence near the lower level of the nudging layers when these are imposed at higher altitudes; these can be reduced by further smoothing the profile of nudging rates and do not affect our conclusions (see Fig. S3 and discussion in supplementary material).

The suppression of vertical EP fluxes seen during the recovery phase of the events in Fig. 1b and in the nudging experiments of HS14 is also reproduced here (Figs. 2e-h). The reduction is strongest in $s 70$ (Fig. $2 \mathrm{~h}$ ), comparable to that seen in the base run, and weakens with the altitude of the imposed anomalies. Significant suppression is still obtained even in $s l$ when the anomalies are imposed near the stratopause. For the week or two around the central date of the imposed warming, particularly in the cases $s 8$ and $s 30$, the negative flux anomalies emerge at successively lower altitudes, corresponding to the descending region of absorption. This indicates at least some of these anomalies arise due to filtering by the mean flow. However, the anomalies in $s 1$ (Fig. 2e) arise synchronously throughout the depth of the stratosphere at layers with no strong mean flow anomalies. This suggests that simple ideas of filtering or reflection of propagating modes (for which flux anomalies would be expected to propagate with a bounded vertical group speed) cannot explain all of these anomalies.

In all cases anomalous fluxes arise within the troposphere below 300hPa. However the magnitude of the anomaly averaged over the timescale for recovery for the vortex is small compared with the time variation that appears in the ensemble mean shown in Figs. 2e-h, and while this time variation seems likely to be internal variability rather than a systematic signal, simple estimates suggest that to verify this convincingly a much larger ensemble would be required. Constraining the details of the time-dependence of the flux anomalies with the troposphere therefore remains a significant challenge.

Figures 2g-h also show that significant enhancement of the upward fluxes are also obtained during the 30 days prior to the central date in $s 70$ and $s 30$, indicating a role for the stratospheric mean state in the amplification of waves responsible for sudden warmings. In contrast to the flux anomalies after the central date in $s 70$ and $s 30$ that are similar in magnitude to the base run composite, the flux anomalies prior to the central date are a factor of 10 weaker to those in the corresponding period in Fig. 1b. This enhancement 
was not obtained by HS14, likely because anomalies were imposed only at the time of the stratospheric wind reversal.

On first sight this result appears to suggest that the stratospheric influence is only responsible for about $10 \%$ of the amplification of the waves prior to the central date. It does firmly establish that the troposphere must be in a favourable state in order for the full amplification to occur, since if the wave amplification was independent of the tropospheric state, it would have been fully recovered in the nudged ensemble. However, this does not quantify the role of the stratospheric state in the amplification of the waves when troposphere is in such a favourable state.

To investigate this role further, an additional pair of ensembles, $m 30$ and $m 20$, are also produced. These are 80-day integrations initialized from the base run 30 and 20 days (respectively) prior to the central date of each of the warmings composited in Fig. 1. They are relaxed towards the time average of the base run $\left(X_{c}\right)$ with the same profile of relaxation rates used by $s 70$. The relaxation is switched on smoothly using $r(t, 5 d)$. By preventing the stratospheric mean state from evoloving with the amplifying waves, these ensembles test for the role of the stratospheric mean state in this amplification.

Figures 3a,b show the difference in the same fluxes shown in Figs.2e-h between the integrations in $m 30$ and $m 20$ and the corresponding periods in the base run, respectively. When the stratospheric mean state is prevented from evolving, this amplification is reduced. When the nudging is switched on 30 days prior to the central date $(\mathrm{m} 30)$, the amplification is weakened by $50 \%$ relative to that seen in the base run (Fig. 1b). The stratospheric constraint must be imposed sufficiently early in the evolution, however; in $m 20$ where the nudging is switched on 20 days prior to the central date relatively weaker suppression of the amplification is seen only in the upper troposphere. We have confirmed that the full amplification seen in Fig. 1b is not a result of chaotic error growth but is in fact due to the stratospheric constraint (see Fig. S4).

The stratospheric mean state does therefore play a significant role in the amplification of the waves responsible for the breakdown of the vortex in these simulations, as sugggested by the theories of Plumb [1981] and Matthewman and Esler [2011] and demonstrated by the model experiments of Scott and Polvani [2004]. On the other hand it is clear from Figs $2 \mathrm{~g}, \mathrm{~h}$ that the troposphere must also be in a favourable state for the 
amplification of the waves to occur. Characterizing the nature of these tropospheric states in detail is beyond the scope of this work.

Returning to Fig. 2, panels (i-l) show the tropospheric wind anomalies in each ensemble. An equatorward shift of the jet persisting until nearly 60 days following the central date is obtained in all cases. The magnitude of the anomalies decrease with the height of the imposed anomalies, with $s 70$ producing anomalies nearly as strong as those in the base run.

Recent work [HS14, Smith and Scott, 2016; Hitchcock and Simpson, 2016] has highlighted the importance of the planetary-scale wave field for communicating the influence of the stratospheric anomalies to the tropospheric jet during the recovery phase of stratospheric sudden warmings. Figure 3c shows the time-averaged upper tropospheric zonal wind anomalies (from $30^{\circ}$ to $40^{\circ} \mathrm{N}$ ) and vertical EP flux anomalies in each ensemble. These quantities are proportional in the forced response. The present experimental design cannot directly attribute the suppressed vertical wave fluxes to the imposed stratospheric anomalies (as opposed to being determined by the evolution of the tropospheric flow); however, as was found by HS14 in a comprehensive model, the vertical EP fluxes are not correlated with the tropospheric jet variability in the base and control runs suggesting the suppressed fluxes determine the jet response, not the reverse.

\section{Conclusions}

We have demonstrated that anomalies associated with stratospheric sudden warmings, even when imposed in the upper stratosphere $(s I)$ within a layer representing only $0.2 \%$ of the mass of the atmosphere, can impart a significant, robust impact on the waves and mean flow below, in both the stratosphere and troposphere. This has been achieved with a mechanistic circulation model through a set of numerical experiments that identifies a deterministic impact by averaging across a large ensemble of integrations, each with a different tropospheric initial condition. The experiments clearly reveal how the response to imposed zonally-symmetric stratospheric anomalies vary with the height $\left(p_{b}\right)$ above which they are imposed.

In all cases we find that the influence of the anomalies extends well below $p_{b}$. In cases where $p_{b}$ lies well above the tropopause, there is a clear, localized region of wave, mean-flow interaction which emerges below the region of nudging when the imposed 
westward anomalies are strongest, producing a descent of the westward anomalies (Fig. 2ad). This signal weakens in cases where $p_{b}$ lies closer to the tropopause, and in no case does it penetrate the tropopause, suggesting that the mechanisms for downward influence within the stratosphere are distinct from those responsible for the suppression of upward wave flux within the troposphere after the warming. One reason this mechanism may be restricted to the stratosphere is the presence of the strong wave guide at the edge of the polar vortex; another reason may be that the coherence of the signal is lost in the presence of strong tropospheric variability.

There is some similarity to the mechanism described by Matsuno [1971] for the evolution of sudden warmings including the downward migration of wind anomalies but it is important to note that our experiments have established that there is genuine downward propagation of information. This contrasts with the Plumb and Semeniuk [2003] characterisation of the Matsuno [1971] mechanism as similar to the Plumb [1977] model of the equatorial quasibiennial oscillation, in which there is no downward propagation of information. Note also that the downward propagation of the zonal flow (and flux divergence) anomalies seen at the onset of the event in Fig. 2a,b is much slower than the downward migration seen in the base run composite (Fig. 1a). Thus, rather as is the case for the role of the stratospheric flow in enhancing upward wave fluxes prior to the warming (see discussion below), the mechanism responsible for the downward propagation may be an important part of the evolution of sudden warmings, but it must be accompanied by other physical effects.

The imposed anomalies suppress vertical fluxes of wave activity throughout the depth of the atmosphere during the recovery phase of the imposed warmings (Fig. 2eh), in agreement with the results of HS14. When the anomalies are imposed in the lower stratosphere, the flux anomalies are as large as those found in the free running integration (Fig. 1b). The flux anomalies weaken as $p_{b}$ is reduced. When the circulation anomalies are imposed in the middle or upper stratosphere, the flux anomalies arise nearly simultaneously throughout the depth of the stratosphere, suggesting the possible relevance of barotropic modes for this coupling.

In all cases an equatorward shift of the tropospheric jet is obtained over much of the recovery period of the imposed warming. The structure of the wind anomalies are only 
weakly dependent on $p_{b}$, but their magnitude reduces as $p_{b}$ reduces, and correlates with the tropospheric upward wave flux anomalies.

When the anomalies are imposed lower in the stratosphere, significant enhancement of the vertical wave fluxes are found prior to the central date of the imposed warming. The amplification in the nudged ensembles is only of the order of $10 \%$ of that in the base run composite. However, when the stratospheric zonal mean is constrained to its time mean state sufficiently early during the onset of the warming, the amplification of the waves is found to be reduced by about $50 \%$. This provides strong and novel evidence in a full primitive-equations model for the coupled evolution of waves and the mean state during the onset of a warming, expected, for example, from the ideas of resonant amplification [Plumb, 1981; Matthewman and Esler, 2011].

This constitutes an important asymmetry in the response, in the sense that imposing the stratospheric anomalies prior to the central date only recovers a fraction of the enhanced upward fluxes of wave activity, while the imposed stratospheric anomalies during the recovery phase are sufficient to produce the full suppression. While the onset of the warmings seem therefore to require appropriate configurations of both the stratosphere and troposphere, the post-warming evolution seems only to require the configuration of the stratospheric state.

These experiments reveal a substantial influence on the tropospheric circulation by the full depth of the stratosphere, indicating clear potential for stratospheric forcings to impact on the surface through the polar vortex. They reveal a variety of distinct mechanisms by which the zonal mean flow and the planetary waves interact to communicate this influence, highlighting in particular the potential for the stratospheric state to affect the evolution of the waves over a deep region of the atmosphere.

\section{A: Temperature Relaxation Profile}

Numerical integrations are performed using a modified version of the Reading Intermediate General Circulation Model (IGCM), version 1. The code integrates the dry hydrostatic primitive equations on the sphere Hoskins and Simmons [1975] and has been modified to use the angular-momentum conserving vertical discretization of Simmons and Burridge [1981] on hybridized pressure levels. The model climate is determined by a linear relaxation towards an equilibrium temperature profile that is convectively stable but 
baroclinically unstable [Held and Suarez, 1994]. All reference quantities below are defined on model levels ( $p$ is determined by setting $p_{s}=p_{0}$ with $p_{0}=1000 \mathrm{hPa}$ ).

The radiative equilibrium temperature profile follows Polvani and Kushner [2002], with several modifications; all notation below follows their definitions. The stratospheric profile is specified by

$$
T_{e q}^{\text {strat }}=T_{U S}^{\prime}(p)+W(\phi) T_{P V}^{\prime}(p)
$$

in which the meridional weighting function $W$ is the same as that used by Polvani and Kushner [2002] but with the vortex in the Northern Hemisphere. The polar vortex profile is specified by

$$
T_{P V}^{\prime}(p)=T_{T}\left(\left(\frac{p}{p_{T}}\right)^{-R \gamma / g}-1\right)
$$

and is lowered by setting $p_{T}$ to $300 \mathrm{hPa}$. The US Standard Atmosphere used outside the polar region is modified by reducing the temperature everywhere $\left(T_{U S}^{\prime}=T_{U S}-16.65 \mathrm{~K}\right)$ so that $T_{U S}^{\prime}\left(p_{T}\right)$ is equal to $200 \mathrm{~K}$ and the tropopause in the equilibrium profile occurs at pressure levels closer to the Earth's tropopause. The hemispheric asymmetry parameter $\epsilon$ used by Polvani and Kushner [2002] is set to $0 \mathrm{~K}$.

The profile of radiative damping timescales (above the boundary layer) is set to

$$
\alpha=\alpha_{T}+\frac{1}{2}\left(\tanh \left(\frac{z-z_{S}}{\sigma_{z}}\right)+1\right)\left(\alpha_{S}-\alpha_{T}\right)
$$

with $\alpha_{T}^{-1}=40 \mathrm{~d}$ and $\alpha_{S}^{-1}=5 \mathrm{~d}$. The $\log$-pressure height $z$ is set to $H \log (p / p 0)$ where $H$ $=7 \mathrm{~km}$, and finally $z_{s}=35 \mathrm{~km}$ and $\sigma_{z}=7 \mathrm{~km}$.

\section{Acknowledgments}

PHi acknowledges support from European Research Council ACCI grant Project No. 267760.

The data from all model integrations are available from the authors upon request.

\section{References}

Albers, J. R., and T. Birner (2014), Vortex preconditioning due to planetary and gravity waves prior to sudden stratospheric warmings, J. Atmos. Sci., 71, 4028-4054, doi: 10.1175/JAS-D-14-0026.1.

Andrews, D. G., J. R. Holton, and C. B. Leovy (1987), Middle Atmosphere Dynamics, Academic Press, London, UK. 
Baldwin, M. P., and T. J. Dunkerton (2001), Stratospheric harbingers of anomalous weather regimes, Science, 294, 581-584, doi:10.1126/science.1063315.

Coughlin, K., and K. K. Tung (2005), Tropospheric wave response to decelerated stratosphere seen as downward propagation in northern annular mode, J. Geophys. Res., 110, D01,103, doi:10.1029/2004JD004661.

Douville, H. (2009), Stratospheric polar vortex influence on Northern Hemisphere winter climate variability, Geophys. Res. Lett., 36, L18,703, doi:10.1029/2009GL039334.

Gray, L. J. (2003), The influence of the equatorial upper stratosphere on stratospheric sudden warmings, Geophys. Res. Lett., 30, 1166, doi:10.1029/2002GL016430.

Gray, L. J., S. Crooks, C. Pascoe, S. Sparrow, and M. Palmer (2004), Solar and QBO influences on the timing of stratospheric sudden warmings, J. Atmos. Sci., 61, 2777-2796.

Hardiman, S. C., and P. H. Haynes (2008), Dynamical sensitivity of the stratospheric circulation and downward influence of upper level perturbations, J. Geophys. Res., 113, D23,103, doi:10.1029/2008JD010168.

Haynes, P. H., C. J. Marks, M. E. McIntyre, T. G. Shepherd, and K. P. Shine (1991), On the "downward control" of extratropical diabatic circulations by eddy-induced mean zonal forces, J. Atmos. Sci., 48, 651-678.

Held, I. M., and M. J. Suarez (1994), A proposal for the intercomparison of the dynamical cores of atmospheric general circulation models, Bull. Amer. Meteor. Soc., 75, 18251830.

Hitchcock, P., and I. R. Simpson (2014), The downward influence of stratospheric sudden warmings, J. Atmos. Sci., 71, 3856-3876, doi:10.1175/JAS-D-14-0012.1.

Hitchcock, P., and I. R. Simpson (2016), Quantifying forcings and feedbacks following stratospheric sudden warmings, J. Atmos. Sci., 73, 3641-3657, doi:10.1175/JAS-D-160056.1 .

Hitchcock, P., T. G. Shepherd, M. Taguchi, S. Yoden, and S. Noguchi (2013a), Lowerstratospheric radiative damping and polar-night jet oscillation events, J. Atmos. Sci., 70, 1391-1408, doi:10.1175/JAS-D-12-0193.1.

Hitchcock, P., T. G. Shepherd, and G. L. Manney (2013b), Statistical characterization of Arctic Polar-night Jet Oscillation events, J. Clim., 26, 2096-2116, doi:10.1175/JCLI-D12-00202.1.

Holton, J. R., and C. Mass (1976), Stratospheric vacillation cycles, J. Atmos. Sci., 33, $2218-2225$. 
Hoskins, B. J., and A. J. Simmons (1975), A multi-layer spectral model and the semiimplicit method, Q. J. R. Meteorol. Soc., 101, 637-655.

Ineson, S., A. A. Scaife, J. R. Knight, J. C. Manners, N. J. Dunstone, L. J. Gray, and J. D. Haigh (2011), Solar forcing of winter climate variability in the Northern Hemisphere, Nat. Geosci., 4, 753-757, doi:10.1038/ngeo1282.

Kodera, K., and Y. Kuroda (2002), Dynamical response to the solar cycle, J. Geophys. Res., 107, 4749, doi:10.1029/2002JD002224.

Manzini, E., A. Y. Karpechko, J. Anstey, M. P. Baldwin, R. X. Black, C. Cagnazzo, N. Calvo, A. Charlton-Perez, B. Christiansen, P. Davini, E. Gerber, M. Giorgetta, L. Gray, S. C. Hardiman, Y.-Y. Lee, D. R. Marsh, B. A. McDaniel, A. Purich, A. A. Scaife, D. Shindell, S.-W. Son, S. Watanabe, and G. Zappa (2014), Northern winter climate change: Assessment of uncertainty in CMIP5 projections related to stratospheretroposphere coupling, J. Geophys. Res., 119, 7979-7998, doi:10.1002/2013JD021403.

Matsuno, T. (1971), A dynamical model of the stratospheric sudden warming, J. Atmos. Sci., 28, 1479-1494.

Matthewman, N. J., and J. G. Esler (2011), Stratospheric sudden warmings as selftuning resonances. part I: Vortex splitting events, J. Atmos. Sci., 68, 2481-2504, doi: 10.1175/JAS-D-11-07.1.

Muthers, S., J. G. Anet, C. C. Raible, S. Brönnimann, E. Rozanov, F. Arfeuille, T. Peter, A. I. Shapiro, J. Beer, F. Steinhilber, Y. Brugnara, , and W. Schmutz (2014), Northern hemispheric winter warming pattern after tropical volcanic eruptions: Sensitivity to the ozone climatology, J. Geophys. Res., 119, 1340âĂŞ1355, doi:10.1002/2013JD020138.

Perlwitz, J., and N. Harnik (2004), Downward coupling between the stratosphere and troposphere: The relative roles of wave and zonal mean processes, J. Clim., 17, 49024909, doi:10.1175/JCLI-3247.1.

Plumb, R. A. (1977), The interaction of two internal waves with the mean flow: Implications for the theory of the quasi-biennial oscillation, J. Atmos. Sci., 34, 1847-1857.

Plumb, R. A. (1981), Instability of the distorted polar night vortex: a theory of stratospheric warmings, J. Atmos. Sci., 38, 2514-2531.

Plumb, R. A., and K. Semeniuk (2003), Downward migration of extratropical zonal wind anomalies, J. Geophys. Res., 108, 4223, doi:10.1029/2002JD002773.

Polvani, L. M., and P. J. Kushner (2002), Tropospheric response to stratospheric perturbations in a relatively simple general circulation model, Geophys. Res. Lett., 29, 1114, 
doi:10.1029/2001GL014284.

Previdi, M., and L. M. Polvani (2014), Climate system response to stratospheric ozone depletion and recovery, Q. J. R. Meteorol. Soc., 140, 2401-2419, doi:10.1002/qj.2330.

Scaife, A. A., A. Y. Karpechko, M. P. Baldwin, A. Brookshaw, A. H. Butler, R. Eade, M. Gordon, C. MacLachlan, N. Martin, N. Dunstone, and D. Smith (2015), Seasonal winter forecasts and the stratosphere, Atmos. Sci. Let., 17, 51-56, doi:10.1002/asl.598.

Scott, R. K., and L. M. Polvani (2004), Stratospheric control of upward wave flux near the tropopause, Geophys. Res. Lett., 31, L02,115.

Shaw, T. A., and J. Perlwitz (2013), The life cycle of northern hemisphere downward wave coupling between the stratosphere and troposphere, J. Clim., 26, 1745-1763, doi: 10.1175/JCLI-D-12-00251.1.

Sigmond, M., J. F. Scinocca, V. V. Kharin, and T. G. Shepherd (2013), Enhanced seasonal forecast skill following stratospheric sudden warmings, Nat. Geosci., 6, 98-102, doi: 10.1038/NGEO1698.

Simmons, A. J., and D. M. Burridge (1981), An energy and angular-momentum conserving vertical finite-difference scheme and hybrid vertical coordinates, Mon. Wea. Rev., $109,758-766$.

Smith, K. L., and R. K. Scott (2016), The role of planetary waves in the tropospheric jet response to stratospheric cooling, Geophys. Res. Lett., 43, 2904âĂŞ2911, doi: 10.1002/2016GL067849. 

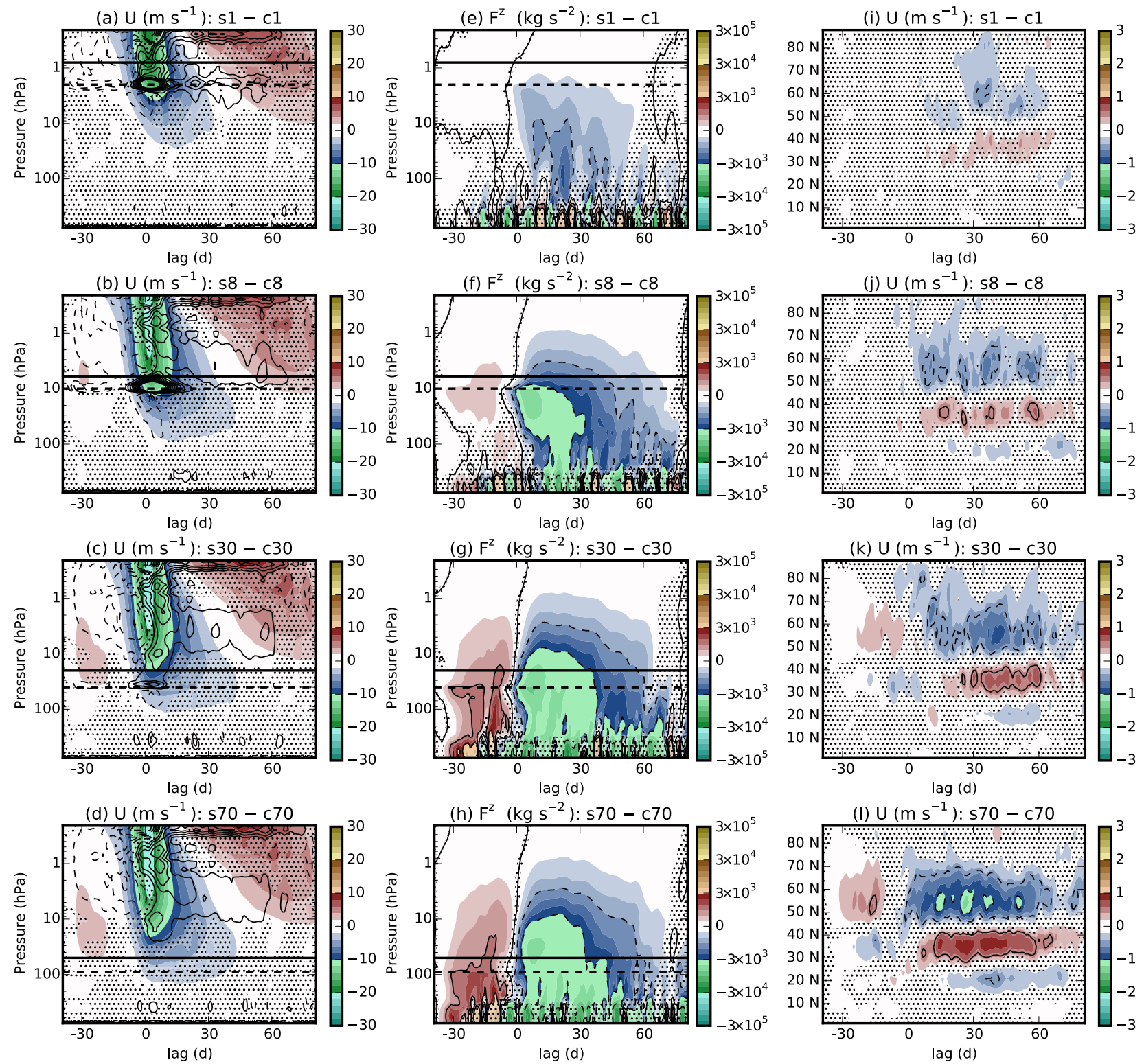

Figure 2. Ensemble mean anomalies of $s 1, s 8, s 30, s 70$ relative to their respective controls for the same quantities shown in Fig. 1. The horizontal lines in panels (a-h) indicate the level at which the nudging is zero (dashed) and full strength (solid). Statistical significance is indicated as in Fig. 1, estimated using a paired-sample t-test. 

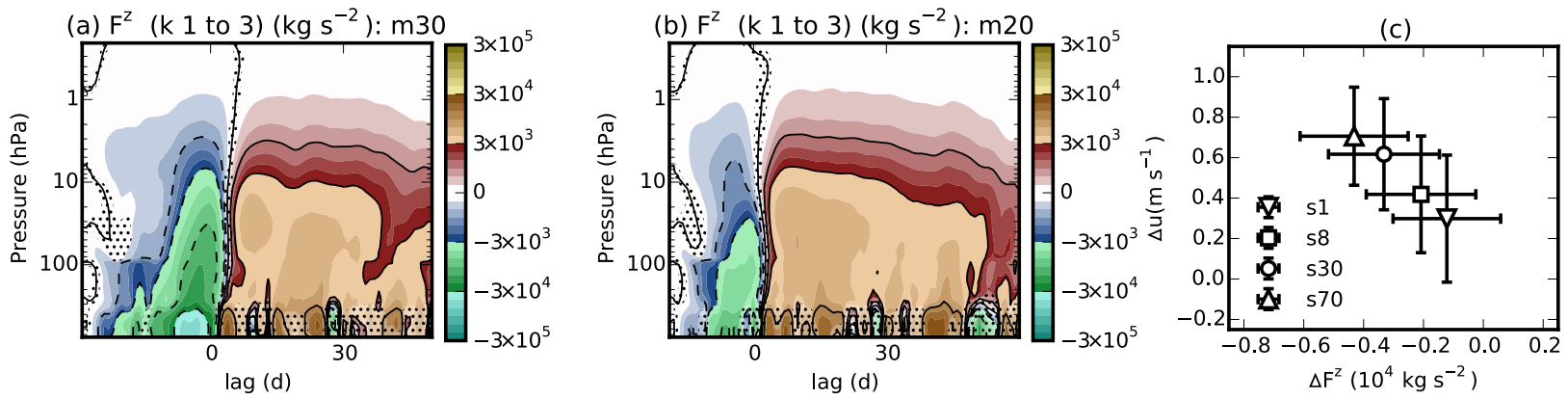

Figure 3. Ensemble mean anomalies from (a) $m 30$ and (b) $m 20$ of the vertical component of the EP flux due to planetary-scale waves, as an anomaly from the corresponding periods in the base run. Statistical significance is computed and indicated as in Fig. 2. (c) Time averaged (days 30-60) zonal mean wind averaged over $30^{\circ}-40^{\circ} \mathrm{N}$ and $500 \mathrm{hPa}$ to $200 \mathrm{hPa}$ plotted against vertical EP flux due to planetary-scale eddies averaged over $50^{\circ}-80^{\circ} \mathrm{N}$ and $500 \mathrm{hPa}$ to $200 \mathrm{hPa}$ from $s 1, s 8, s 30, s 70$. Confidence intervals at the $95 \%$ level are indicated for each quantity. 


\title{
Supporting Information for
}

\section{"Stratospheric control of planetary waves"}

\author{
Peter Hitchcock $^{1 *}$ and Peter H. Haynes ${ }^{1}$ \\ ${ }^{1}$ Department of Applied Mathematics and Theoretical Physics, University of Cambridge, Cambridge, UK.
}

\section{Contents}

1. Text $\mathrm{S} 1$ to $\mathrm{S} 5$

2. Figures $\mathrm{S} 1$ to $\mathrm{S} 5$

\section{Introduction}

\section{Text S1.}

Supplementary Figure 1 shows the climatology (time mean) of the zonal mean, zonal winds in each of the control runs $c 1, c 8, c 30$, and $c 70$. The differences between these climatologies and the base run climatology are shown by the contour lines with an interval of $0.5 \mathrm{~m} \mathrm{~s}^{-1}$. This demonstrates that the effect of nudging the zonally symmetric component of the stratosphere to the climatological state of the base run has a minimal effect on the zonal mean basic state. The impact of the stratospheric nudging on the Northern Hemisphere troposphere amounts to less than a $0.25 \mathrm{~m} \mathrm{~s}^{-1}$ change in the tropospheric winds in all cases but $c 70$, where a dipolar anomaly of approximately $0.5 \mathrm{~m}-1$ corresponding roughly to a poleward shift of the jet. The changes are small relative to the internal variability of the winds; it is therefore unlikely that these changes will have a significant effect on the response of the system to the imposed anomalies. The fact that the tropospheric response seen in Fig. 21 closely resembles the composite response shown in Fig. 1c also confirms this claim.

\section{Text S2.}

Supplementary Figure 2a shows the vertical profile of the mid-latitude average of the standard deviation of the zonal mean zonal wind in the base run and in each of the con-

\footnotetext{
${ }^{*}$ Current address, National Center for Atmospheric Research, 3450 Mitchell Lane, Boulder, CO, USA

Corresponding author: Peter Hitchcock, phitch@ucar .edu
} 
trol runs, plus one additional control run, $c 100$, with $p_{b}=200 \mathrm{hPa}$ and $p_{t}=80 \mathrm{hPa}$. The boundary of the nudging layer for each case is indicated by the colored lines. Within the nudging layer where the relaxation is at its full strength, the internal variability is strongly suppressed; the variability is reduced to a lesser degree even below $p_{b}$. To some extent this need not indicate anything artificial - if variability in the stratosphere is driving some component of variability in the tropospheric flow as our experiments have demonstrate, eliminating the stratospheric variability should remove this component from the tropospheric variability as well. Nonetheless this figure demonstrates that the nudging layer cannot be moved much below $90 \mathrm{hPa}$ without substantially constraining the tropospheric flow. Figure S3b shows the same quantity but computed as the ensemble spread over the nudged runs relative to their respective control runs, averaged over days 15 to 60 following the central date. This spread agrees closely with the internal variability.

\section{Text S3.}

Supplementary Figure 3 shows plots equivalent to those in Fig. 2 but for two additional ensembles, $s 8 w 6 h$ and $s 8 w 1 d$, nudged with alternative profiles of the relaxational timescale, specified using different values for the parameters in (2). In both cases $q=4$, $p_{b}=10 \mathrm{hPa}$ and $p_{t}=3 \mathrm{hPa}$. The first, $s 8 w 6 h$, is an ensemble of 600 integrations with $\tau_{1}=6 \mathrm{~h}$ (Fig. S2a,c,e), while second, $s 8 w 1 d$, is an ensemble of 400 integrations with $\tau_{1}$ $=1 \mathrm{~d}$ (Fig. S2b,d,f). Differences in both cases are taken from a control run relaxed to $X_{c}$ with the corresponding nudging profile, but in the latter case the differences are taken from the time mean the control run, so they do not vanish at the onset of the integrations. These ensembles may be seen as corresponding to a profile intermediate between $s l$ and $s 8$, though we discuss these plots relative to the latter.

In both cases the EP flux convergence near $p_{b}$ prior to the central date seen in Fig. $2 \mathrm{~b}$ is no longer present. The strong anomalous divergence around the central date in Fig. $2 \mathrm{~b}$ is reduced in these ensembles, and is weaker in $s 8 w 1 d$, consistent with the weaker vertical shear induced by the nudging. The descending region of anomalous convergence is still present, though again is somewhat weaker than in Fig. 2b. Nonetheless, the weakened vertical fluxes throughout the depth of the stratosphere, and the shift of the tropospheric jet seen in Fig. 2 are also present in these alternative ensembles; the mean jet shift in both cases is consistent with the uncertainty shown in Fig. 3c. 
We conclude from these further integrations that the EP flux artifacts near $p_{b}$ are not playing a significant role in the evolution of the flow below the nudging layer.

\section{Text S4.}

Supplementary Figure 4 demonstrates that the amplification of the waves prior to stratospheric sudden warmings is fully recovered when the base run is restarted $30(\mathrm{~m} 30 \mathrm{c})$ or $20(\mathrm{~m} 20 \mathrm{c})$ days prior to the events. The panels show the evolution of the vertical EP flux in ensembles equivalent to $m 30$ and $m 20$ but with no stratospheric constraint, an as an anomaly from the evolution of the base run over the same period. Because the restarts are based on instantaneous output at a single timestep, the full information required by the leap-frog timestep used by the model to reproduce bit-for-bit evolution of the runs is not available, and this leads ultimately to diverging trajectories. However, this error growth only becomes significant well after the onset of the stratospheric event. This confirms that the effect demonstrated in Fig. 3a,b is in fact due to constrained stratospheric winds, not due to chaotic error growth.

\section{Text S5.}

Supplementary Figure 5 shows the effects of modifying the profile of the linear relaxation on the suppression of the wave amplification shown in Figs. 3ab. In each case an ensemble similar to $m 30$ or $m 20$ has been carried out. Panels a and b correspond directly to Figs. $3 \mathrm{a}$ and $\mathrm{b}$ but for the nudging profile in (2) modified by setting $\tau_{0}=1 \mathrm{~d}$ and $q$ $=4 ; p_{b}$ is set to $90 \mathrm{hPa}$ and $p_{t}$ to $30 \mathrm{hPa}$. The weaker nudging strength has the expected effect of allowing for more amplification of the wave fluxes. Panel c shows an ensemble equivalent to $m 30$ but with $\tau_{0}=1 \mathrm{~d}$ and $q=4 ; p_{b}$ set to $200 \mathrm{hPa}$ and $p_{t}$ to $80 \mathrm{hPa}$. Constraining the flow lower in the atmosphere has the effect of reducing the amplification of the wave fluxes. 
(a) $U(\mathrm{~m} / \mathrm{s}): \mathrm{cl}$

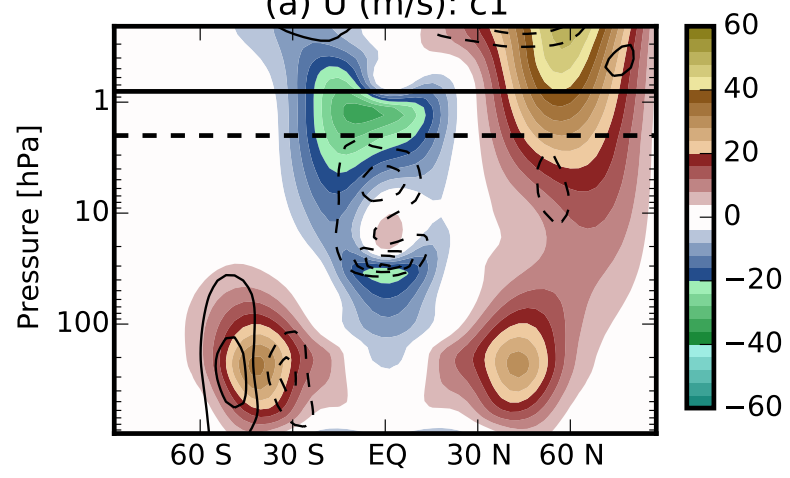

(c) $U(\mathrm{~m} / \mathrm{s}): \mathrm{c} 30$

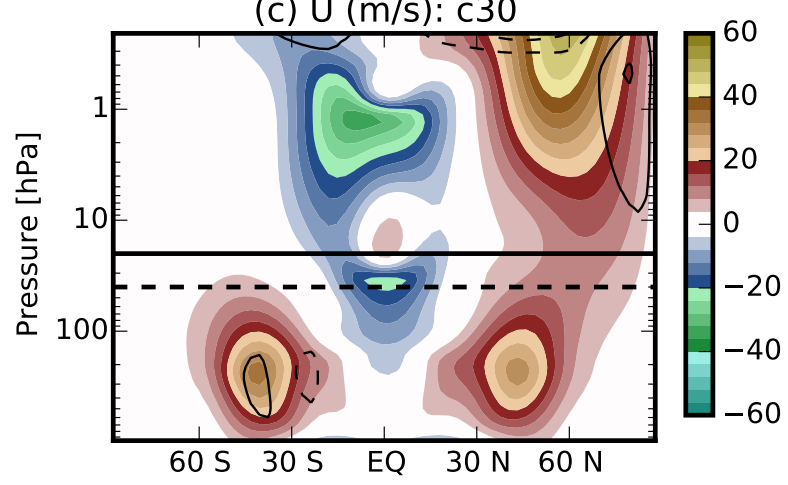

(b) $\cup(\mathrm{m} / \mathrm{s}): \mathrm{c} 8$

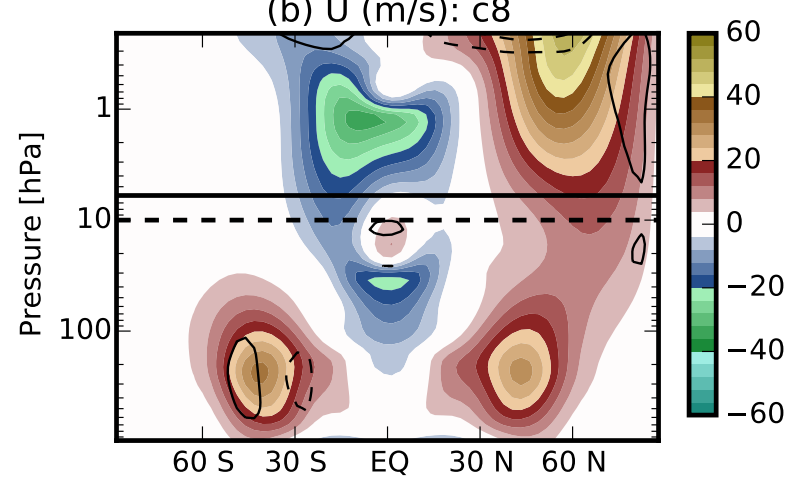

(d) $U(\mathrm{~m} / \mathrm{s}): \mathrm{c} 70$

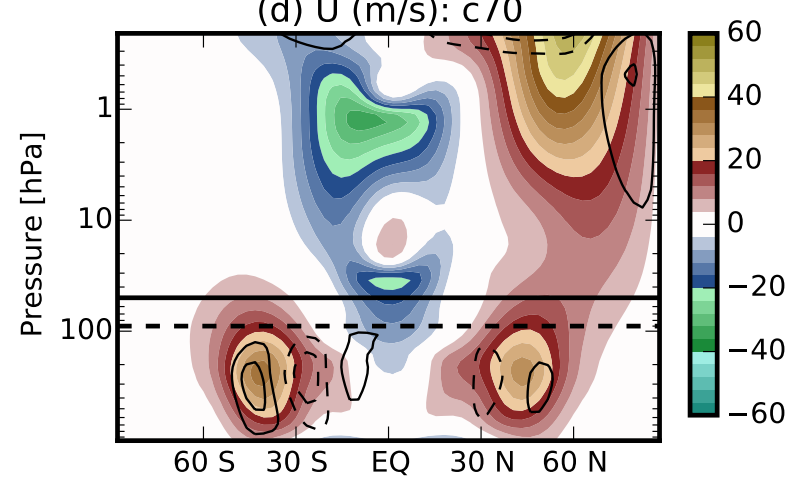

Figure 1. The colored contours show climatological (time mean) zonal mean zonal winds from each of the four control runs. Differences between these climatologies and that of the base run are indicated by the contour lines, shown at intervals of $0.5 \mathrm{~m} \mathrm{~s}^{-1}$. The zero contour is omitted. The horizontal lines indicate the nudging layer in each control run as in Fig. 2. 
(a)

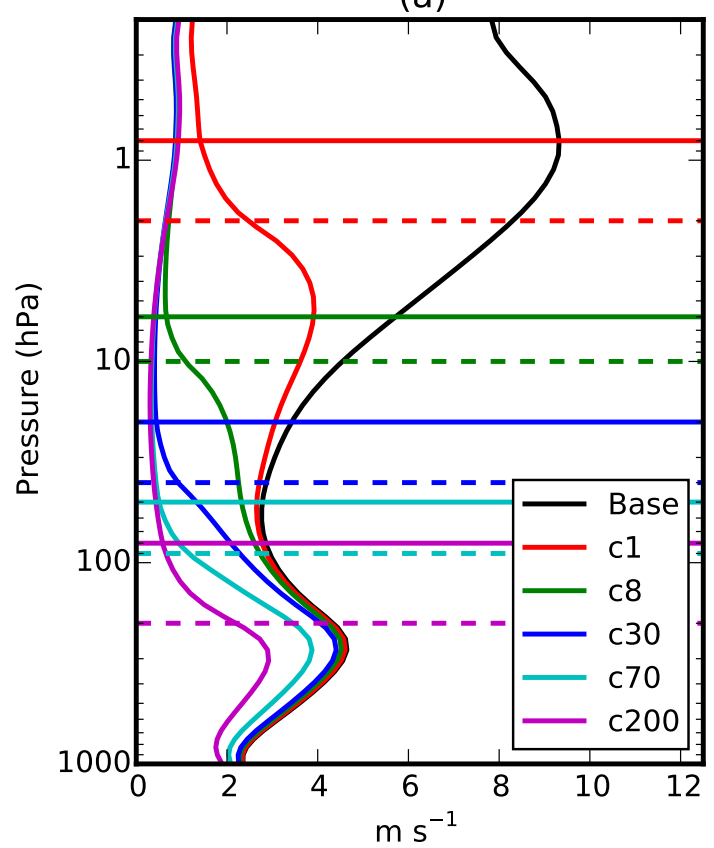

(b)

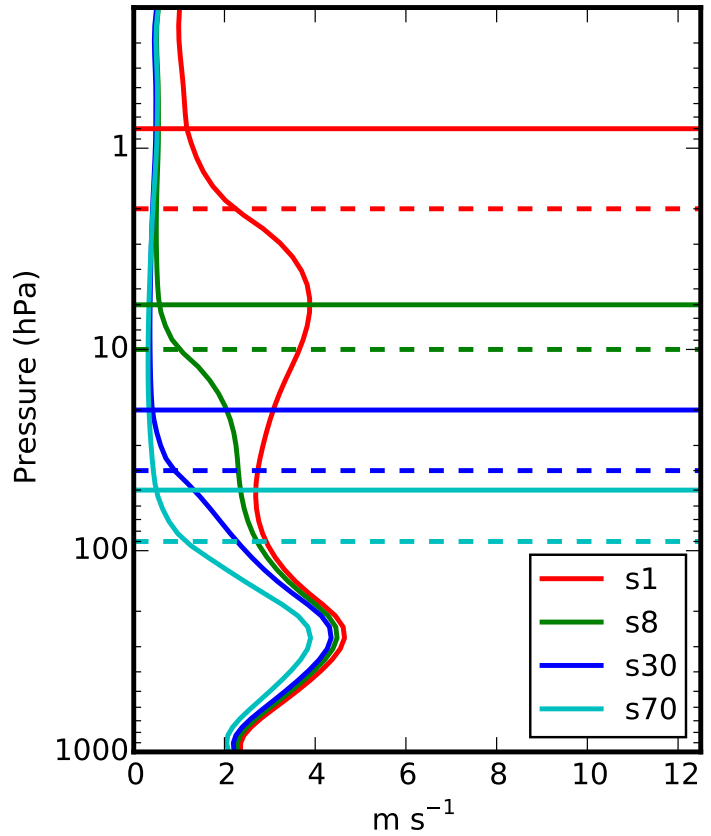

Figure 2. Vertical profile of the mid-latitude $\left(30-60^{\circ} \mathrm{N}\right)$ average standard deviation of zonal mean zonal wind for (a) the internal variability of the control runs and (b) the ensemble spread of the nudged runs, relative to their respective controls. The internal variablity of the base run is also shown in (a). For the nudged runs, the corresponding colored horizontal lines indicate the nudging layer in each control run as in Fig. 2.
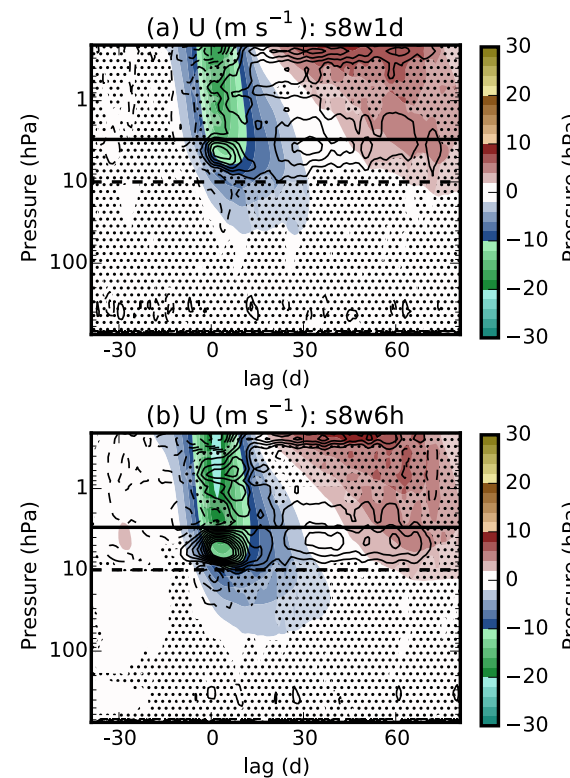
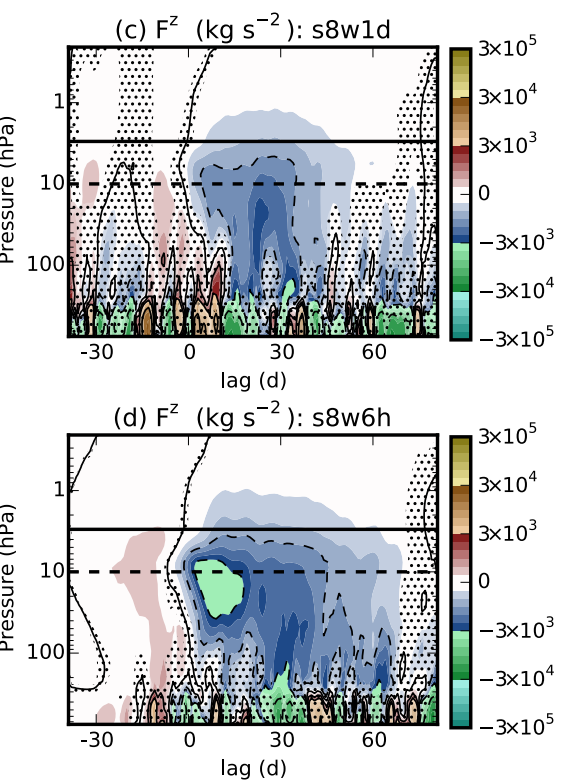
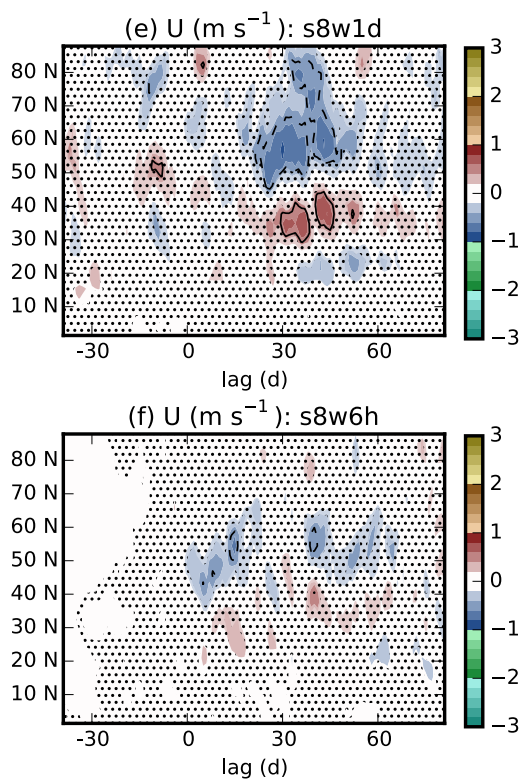

Figure 3. Equivalent to Fig. 2b,f,i but using two alternate profiles of relaxation timescales. See supplementary text S3 for full description. 

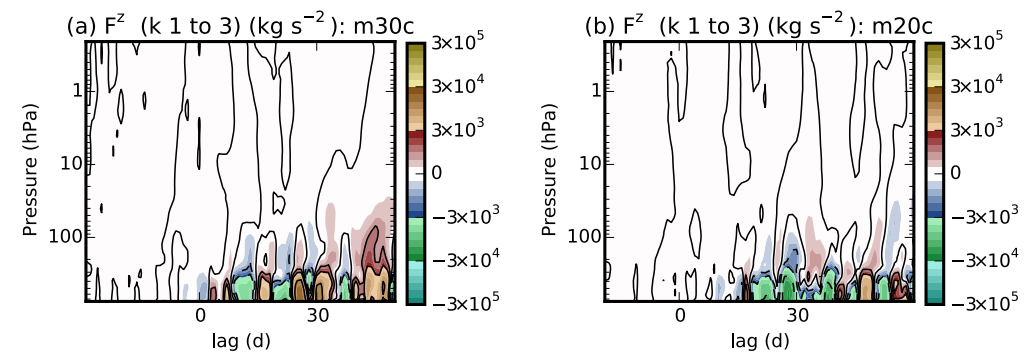

Figure 4. Evolution of the vertical EP flux in a control ensemble initialized from the base run (a) 30 days prior and (b) 20 days prior to the first four hundred sudden warming events. The fluxes are shown as an anomaly relative to the base run over the same periods.
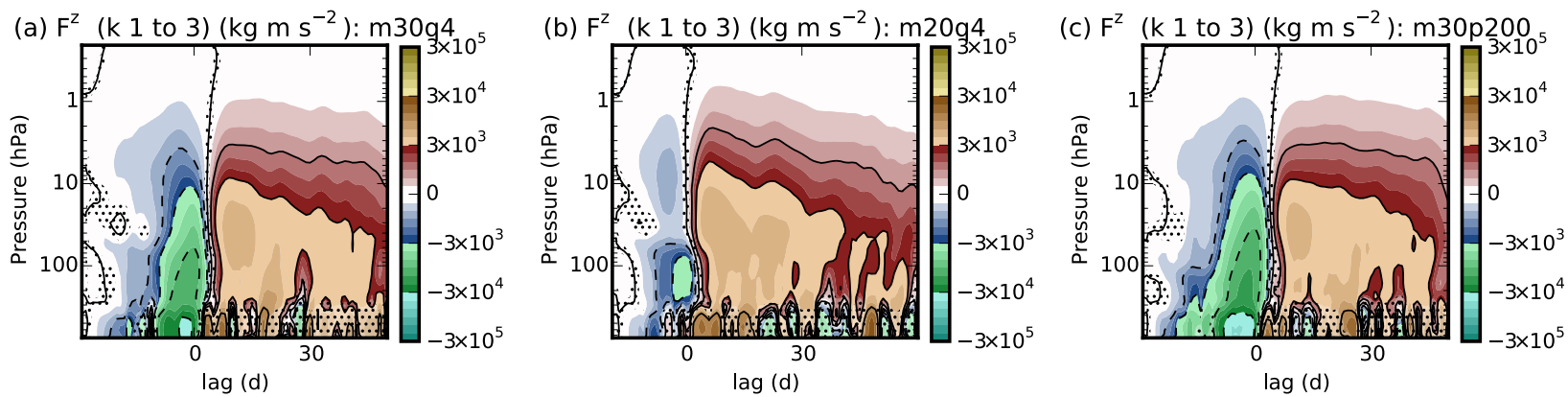

Figure 5. Equivalent to Fig. 3a,b. but for alternative nudging configurations. See text S5 for full description. 